\title{
Comercio interregional de bienes manufacturados en los países de la Alianza del Pacífico desde la Teoría de Linder
}

\author{
WENDY OVANDO ALDANA* \\ Rosa Azalea Canales García** \\ Gabriela Munguía VÁZQueZ***
}

Artículo recibido: 17 de mayo de 2016

Artículo aceptado: 8 de marzo de 2017

Doi: http://dx.doi.org/10.12804/revistas.urosario.edu.co/desafios/a.4876

Para citar este artículo: Ovando Aldana, W., Canales García, R. A., Munguía Vázquez, G. Comercio interregional de bienes manufacturados en los países de la Alianza del Pacífico desde la Teoría de Linder. Desafíos, 29(2). 169-197. doi: http:/ / dx.doi.org/10.12804/revistas. urosario.edu.co/desafios/a.4876

\section{Resumen}

Derivadas de la restructuración económica de finales del siglo XX, la lógica del mercado y la apertura comercial se ban convertido en los ejes rectores del sistema capitalista. La disminución de las barreras al comercio internacionaly los acuerdos comerciales son mecanismos que permiten la promoción del libre comercio. La Alianza del Pacífico

\footnotetext{
* Profesora de tiempo completo de la Facultad de Economía, Universidad Autónoma del Estado de México. Toluca, México. Correo electrónico: wovandoa@uaemex.mx. ORCID: http://orcid.org/0000-0003-0793-6971

** Profesora de tiempo completo de la Facultad de Economía, Universidad Autónoma del Estado de México. Toluca, México. Correo electrónico: azalea_canales@hotmail.com. ORCID: http//:orcid.org/0000-0001-5206-3503

*** Profesora de tiempo completo de la Facultad de Economía, Universidad Autónoma del Estado de México. Toluca, México. Correo electrónico: gmunguia2000@hotmail.com. ORCID: http://orcid.org/0000-0002-3753-8141
} 
representa una iniciativa de integración regional de reciente creación y a cinco años de su firma es necesaria una evaluación. El objetivo de esta investigación es analizar la evolución del comercio de bienes manufacturados entre las naciones de la Alianza del Pacifico. Para alcanzar dicho objetivo, esta investigación se basa en la Teoría de Linder que establece que el comercio de bienes manufacturados será más intensivo entre paises de similar ingreso per cápita. Se realizó un estudio descriptivo con datos de la United Nations Conference on Trade and Development (UNCTAD) y posteriormente se empleó un modelo gravitacional modificado. Con base en la evidencia empirica se establece que el comercio de bienes manufacturados entre las naciones de la Alianza del Pacifico es marginal. Los paises signatarios presentan mayor comercio con otras naciones, principalmente Estados Unidos y China, aun cuando las distancias geográficas con estos países son mayores.

Palabras clave: Alianza del Pacífico, bienes manufacturados, Teoría de Linder, modelo gravitacional

\title{
Interregional Trade in Manufactured Goods in the Pacific Alliance Countries according to Linder's Theory
}

\begin{abstract}
Derived from the economic restructuring of the late twentieth century, the logic of the market and trade liberalization have become the guiding principles of the capitalist system. The reduction of barriers to international trade and trade agreements are mechanisms for promoting free trade. The Pacific Alliance is a regional integration initiative recently created and with five years of existence, a preliminary evaluation is in order. The objective of this article is to analyze the evolution of trade in manufactured goods between the member-countries of the Pacific Alliance. To achieve this objective, the article is based on the theory of Linder which stipulates that trade in manufactured goods will be intensive among countries with similarper-capita income. A descriptive study is conducted with data from UNCT AD and a modified gravity model is used subsequently. Based on the empirical evidence, it is established that trade in manufactured goods between the member-countries of the Pacific Alliance is in fact low. However, the member-countries of the Alliance have increased trade with
\end{abstract}


other countries, mainly with the United States and China, even if the geographical distance with these countries is wider.

Keywords: Pacific Alliance, manufactured goods, Linder's theory, gravity model

\title{
Comércio inter-regional de bens manufaturados nos países da Aliança do Pacífico desde a Teoria de Linder
}

\begin{abstract}
Resumo
Derivado da reestruturação económica de finais do século XX, a lógica do mercado e a apertura comercial se têm convertido nos eixos reitores do sistema capitalista. A diminuição das barreiras ao comércio internacional e os acordos comerciais são mecanismos que permitem a promoção do livre comércio. A Aliança do Pacífico representa uma iniciativa de integração regional de recente criação e após cinco anos da sua assinatura é necessária uma avaliação. O objetivo desta pesquisa é analisar a evolução do comércio de bens manufaturados entre as nações da Aliança do Pacífico. Para alcançar dito objetivo esta pesquisa baseia-se na Teoria de Linder que estabelece que o comércio de bens manufaturados será mais intensivo entre países de similar ingresso per cápita. Realiza-se um estudo descritivo com dados da United Nations Conference on Trade and Development (UNCTAD) eposteriormente empregou-se um modelo gravitacional modificado com base na evidência empírica se estabelece que o comércio de bens manufaturados entre as nações da Aliança do Pacífico marginal. Os países signatários apresentam maior comércio com outras nações, principalmente Estados Unidos e China, ainda que e quando as distâncias geográficas com estes países são maiores.
\end{abstract}

Palavras-chave: Aliança do Pacifico, bens manufaturados, Teoria de Linder, modelo gravitacional

\section{Introducción}

Durante los años setenta del siglo XX, las tasas de ganancia y productividad disminuían y, al mismo tiempo, la esfera financiera predominaba por encima del capital productivo. Aunado a lo anterior, 
la contracción del consumo impactaba al patrón de acumulación de producción rígido (Harvey, 1998; Antunes, 2005; Brenner, 2009). El siguiente paso era reestructurar a las economías capitalistas. De hecho, la restructuración del sistema capitalista comenzó en los países desarrollados con las ideas neoliberales de las administraciones Thatcher y Reagan (Antunes, 2005; Harvey, 2007).

América Latina no quedó exenta de este ajuste económico. Una serie de medidas se plasmaron en el Consenso de Washington, cuyo principal objetivo era el equilibrio de las variables macroeconómicas y la disminución del estado de bienestar. De acuerdo a este Consenso, la apertura económica estaba relacionada con acciones encaminadas a promover, permitir y facilitar el comercio internacional, ya que este último impulsaría el crecimiento económico (Martínez y Soto, 2012). Una de estas acciones la constituyen los acuerdos y tratados comerciales que permitirían a los productos nacionales su inserción en otros mercados internacionales (Solís, 2000). De hecho, los acuerdos comerciales regionales representan una forma de liberar el comercio, en estos hay un compromiso por reducir las barreras al comercio, exclusivamente, entre los países signatarios (Carbaugh, 2004).

La Alianza del Pacífico representa una iniciativa de integración regional de reciente creación. En esta propuesta, México, al igual que el resto de los países miembro (Chile, Colombia y Perú) se proponen como uno de sus objetivos impulsar no solo el crecimiento, sino el desarrollo económico. En cuestiones de comercio internacional buscan "Construir, de manera participativa y consensuada, un área de integración profunda para avanzar progresivamente hacia la libre circulación de bienes, servicios, capitales, personas y economía" (Alianza del Pacífico, 2016, s/p).

La importancia de esta alianza radica en lo que representan sus economías en conjunto para América Latina: $38 \%$ del producto interno bruto (PIB) de la región, más de $50 \%$ de las exportaciones e importaciones de bienes, 43,5 \% de la Inversión Extranjera Directa (IED), así como 35,6 \% de la población total, en 2014 (Alianza del 
Pacífico, 2016; United Nations Conference on Trade and Development [UNCTAD], 2016).

La Alianza del Pacífico se creó oficialmente en 2011 y a cinco años de su firma es necesario reconocer sus avances y/o retrocesos. En materia comercial, se requiere indagar acerca de los avances en materia de la libre circulación de bienes y servicios. Analizar los bienes manufacturados o productos relacionados con la industria manufacturera es parte del objetivo de este documento debido a que configuran bienes cuya capacidad impulsa, efectivamente, el crecimiento económico (Organización de las Naciones Unidas para el Desarrollo Industrial [ONUDI], 2013). En este ámbito, la industria manufacturera se ha caracterizado por ser un determinante del crecimiento económico general de una economía (Bernat, 1996).

Debido a lo anterior, el objetivo de esta investigación es analizar la evolución del comercio de bienes manufacturados entre las naciones de la Alianza del Pacífico. Lo anterior para determinar el grado de avance en la integración de las economías de los países signatarios.

Para tal efecto, se toma como referencia la Teoría del Comercio Internacional de Linder. Esta establece que el comercio internacional de bienes manufacturados será más intensivo entre los países con un ingreso per cápita similar, en comparación con los países que cuentan con un nivel de ingreso per cápita diferente (Appleyard, Field y Cobb, 2010).

Asimismo, es importante señalar que se realiza un análisis descriptivo del comercio entre naciones a través de porcentajes de participación de las exportaciones e importaciones entre los miembros de la Alianza del Pacífico. Se emplean datos de la UNCTAD y de la Organización Mundial del Comercio (OMC). La evidencia empírica permite establecer que existe una mayor asociación comercial con otras economías y no entre los países signatarios. Para complementar la información se empleó un modelo gravitacional que se centra en la interacción entre la resistencia (distancia geográfica) y la atracción (patrones similares de demanda) (Appleyard, Field y Cobb, 2010). 
La relevancia de este estudio versa en la presentación de un enfoque novedoso para estudiar a la Alianza del Pacífico.

Es importante señalar que una de las limitantes de este estudio radica en que se centra en el estudio de los bienes manufacturados que se comercializan entre los países de la Alianza del Pacífico. Es decir, no se contemplan las transacciones económicas derivadas del comercio de servicios.

Este documento se divide en cinco secciones. En la primera, se hace referencia a la Teoría de Linder y el uso de modelos gravitacionales en los ámbitos teórico y empírico. En la segunda, se presentan los antecedentes, datos generales de la Alianza del Pacífico y la evolución del comercio de bienes manufacturados entre las naciones signatarias. En la tercera, se presenta el modelo gravitacional y su metodología. En la cuarta, se muestran los resultados. En la quinta, se describen las principales conclusiones.

\section{La Teoría del Comercio Internacional de Linder}

La revisión de las teorías del comercio internacional es indispensable para poder comprender la del Comercio Internacional de Linder que se ubica dentro de los enfoques posteriores al modelo de HecksherOhlin (Appleyard, Field y Cobb, 2010).

En el contexto histórico, los teóricos pioneros del comercio internacional se relacionan con los mercantilistas. Durante el siglo XVIII el término mercantilismo se empleó para explicar una serie de ideas económicas que prevalecieron durante los siglos XVI y XVIII (Ekelund y Hébert, 2005). De hecho, no se les considera como una escuela de pensamiento económico sino como una serie de actitudes ante la economía nacional y el comercio internacional (Appleyard, Field y Cobb, 2010). Esta corriente señalaba que una nación debía contar con un comercio exterior estable y sólido. Para ellos, la balanza comercial debía ser superavitaria mediante acumulación de la mayor cantidad posible de oro, por lo que la participación del Estado era esencial para evitar la salida del preciado metal. Algunas medidas de protección al 
comercio se observan durante esta época: aranceles, cuotas y otras formas de política comercial. Una de las principales razones por las cuales fueron criticados los mercantilistas era la referida a que no todas las naciones se verían favorecidas por el comercio internacional. Adam Smith trataría de cubrir este vacío con su propuesta acerca de la ventaja absoluta (Carbaugh, 2004).

Con la idea de la especialización, Smith consideraba que todas las naciones se verían beneficiadas por el comercio internacional (Carbaugh, 2004). Los países deben especializarse en aquellos bienes en los cuales tengan una ventaja absoluta para poder exportarlos, e importar aquellos productos en los cuales sus contrapartes tuvieran una ventaja absoluta (Appleyard, Field y Cobb, 2010). No obstante, Adam Smith no contempló las diferencias en el nivel de desarrollo de las naciones (por ejemplo, comercio entre un país desarrollado y uno en desarrollo). Para resolver este problema, David Ricardo generó el principio de la ventaja comparativa (Carbaugh, 2004; Tan y Zeng, 2014).

La ventaja comparativa señala que la nación que cuenta con ventaja absoluta en todos los bienes que produce debe especializarse en aquel bien que produce con mayor eficiencia; y el país con desventaja absoluta, debe producir aquel bien donde cuenta con una menor desventaja absoluta (Appleyard, Field y Cobb, 2010; Raffo, 2012). En otras palabras, el comercio internacional se puede llevar a cabo entre países de diferente nivel de desarrollo (Carbaugh, 2004; Raffo, 2012). Sin embargo, David Ricardo solo explica que las ventajas dependen de la productividad del trabajo y no el porqué de estas diferencias (Carbaugh, 2004).

Posteriormente, a inicios del siglo XX, dos economistas, Heckscher y Ohlin llenarían este hueco teórico a través de las diferencias en la dotación de factores de la producción (Carbaugh, 2004; Raffo, 2012).

El modelo de Heckscher-Ohlin, considerado como parte de la teoría neoclásica del comercio internacional y también conocido como teoría de la dotación de factores, expresa que las diferentes economías 
exportarán aquellos bienes en los que cuenten con factores de la producción baratos y abundantes (Tan y Zeng, 2014). Por lo tanto, las condiciones de cada nación, relacionadas con la dotación de factores (tierra, trabajo y capital) explicarían las ventajas comparativas (Carbaugh, 2004). Pese a la explicación del modelo Heckscher-Ohlin sobre la dotación de factores de la producción en las naciones, surgieron nuevas teorías del comercio internacional que relajaban sus supuestos. Algunas de las vertientes post Heckscher-Ohlin tratan las diferencias de la tecnología entre las naciones (The Imitation Lag Hypothesis, The Product Cycle Theory), la competencia imperfecta (The Krugman Mode), o bien, las condiciones de la demanda (The Linder Theory) (Appleyard, Field y Cobb, 2010).

Uno de los nuevos enfoques que explica el actual comercio entre las economías que conforman la Alianza del Pacifico es la Teoría de Linder. La referida es pertinente con el estudio de las economías en desarrollo (McPherson, Redfearn y Tieslau, 2001; Bukhari, et al., 2005), no así el modelo Heckscher-Ohlin que consideraba el pleno empleo, la perfecta movilidad de factores e idéntica tecnología entre los países comerciantes (Bukhari, et al., 2005)

La Teoría de Linder supone que el comercio internacional de bienes manufacturados será más intensivo entre los países con un ingreso per cápita similar en comparación con las naciones que cuentan con un nivel de ingreso per cápita diferente (Appleyard, Field y Cobb, 2010). A diferencia del modelo Heckscher-Ohlin, el cual se concentra en el lado de la oferta (factores de la producción), Linder se ocupa de la demanda. Considera que los gustos de los consumidores (demanda) están condicionados por su nivel de ingreso (Appleyard, Field y Cobb, 2010). Es decir, la demanda de bienes manufacturados entre las naciones de la Alianza del Pacífico será alta si cuentan con similares niveles de ingreso per cápita.

Existen diversos estudios que han probado la Teoría de Linder entre diferentes economías en desarrollo, hallando un comercio más intensivo entre países de similar nivel de ingreso (McPherson, Redfearn y Tieslau, 2001; Choi, 2002; Bukhari, et al., 2005). Igualmente, la 
evidencia empírica proveniente de otras investigaciones refiere que no se ha encontrado suficiente sustento a la hipótesis central de Linder (Chow, Kellman y Shachmurove, 1999), o bien, que se puede incorporar su análisis en países desarrollados con un resultado favorable a la Teoría (Arnon y Weinblatt, 1998).

Una forma generalizada de probar la hipótesis de la Teoría de Linder (el comercio internacional de bienes manufacturados será más intensivo entre los países con un ingreso per cápita similar) es a través del modelo gravitacional (Chow, Kellman y Shachmurove, 1999; Arnon y Weinblatt, 1998; McPherson, Redfearn y Tieslau, 2001; Choi, 2002; Bukhari, et al., 2005; Appleyard, Field y Cobb, 2010). Aunque es preciso mencionar que el modelo gravitacional original debe modificarse para emplearlo como una técnica acorde con la Teoría de Linder, lo cual se explicará en el apartado de metodología.

Como se puede observar, la Teoría de Linder permite dar sustento teórico al estudio de las naciones que conforman la Alianza del Pacífico debido a su armonía con los países en desarrollo. Asimismo, se ha identificado al modelo gravitacional como técnica adecuada para probar la teoría mencionada.

En la siguiente sección se identifican los antecedentes de la Alianza del Pacífico y la evolución del comercio de bienes manufacturados entre las naciones de la Alianza, con lo que se determinará si se ha logrado avanzar en la integración de las economías de los países signatarios.

\section{Evolución del comercio de los países de la Alianza del Pacífico}

Durante los años setenta del siglo XX, dentro de las economías capitalistas, las tasas de ganancia y productividad disminuían y, al mismo tiempo, la esfera financiera predominaba por encima del capital productivo. Además, la contracción del consumo impactaba el patrón de acumulación de producción rígido (Harvey, 1998; Antunes, 2005; Brenner, 2009). El siguiente paso era reestructurar al sistema capitalista. De hecho, su restructuración comenzó en los 
países desarrollados con las ideas neoliberales de las administraciones Thatcher y Reagan (Antunes, 2005; Harvey, 2007). El gobierno británico, mediante política de ingresos, trató de controlar la inflación, aumentar la productividad y mantener el pleno empleo (Whiteside, 2000). Paralelamente, se enfrentó a los sindicatos, deshabilitó el estado de bienestar, privatizó las empresas públicas y desmanteló formas de solidaridad social en favor del individualismo y la propiedad privada. En Estados Unidos, hubo recortes presupuestarios, disminución en los impuestos y, de igual manera, se atacó el poder de los sindicatos (Harvey, 2007).

América Latina no quedó exenta de este ajuste económico. Una serie de medidas se plasmó en el Consenso de Washington cuyo principal objetivo era el equilibrio de las variables macroeconómicas y la disminución del estado de bienestar. La base del nuevo modelo económico se cimentaba en el arribo del neoliberalismo que caracteriza la nueva política económica relacionada con el libre mercado y la apertura comercial (Castillo, 2009). De acuerdo a este Consenso, la apertura económica estaba relacionada con acciones encaminadas a promover, permitir y facilitar el comercio internacional, ya que este último impulsaría el crecimiento económico (Martínez y Soto, 2012). Una de estas acciones la constituyen los acuerdos comerciales que permiten a los productos nacionales su inserción en otros mercados internacionales (Solís, 2000). Bajo esta panorámica, los acuerdos comerciales regionales representan una forma de liberar el comercio debido al compromiso de reducir las barreras comerciales entre los países signatarios (Carbaugh, 2004).

En América Latina se observan diferentes tipos de regionalismos que corresponden a etapas históricas disímiles, por ejemplo: el regionalismo cerrado, fuertemente relacionado con el modelo de Sustitución de Importaciones (MSI); el abierto (flexible), afín al periodo de apertura comercial basado en el Consenso de Washington (Van Klaveren, 1997; Sánchez, 1999; Duarte, González y Montoya, 2014), o bien, el posneoliberal, posterior a los años noventa (Sanahuja, 2007; Falomir, 2013). 
Concretamente, la Alianza del Pacífico representa una iniciativa de integración regional de reciente creación, con características del regionalismo abierto porque no solo busca un acuerdo comercial sino un acuerdo multidimensional (Falomir, 2013). En esta propuesta, los países signatarios - Chile, Colombia, México y Perú- se plantean impulsar el crecimiento y el desarrollo económico. En cuestiones de comercio internacional buscan "Construir, de manera participativa y consensuada, un área de integración profunda para avanzar progresivamente hacia la libre circulación de bienes, servicios, capitales, personas y economía” (Alianza del Pacífico, 2016, s/p).

La integración regional Alianza del Pacífico (AP) tiene sus antecedentes en la iniciativa Arco del Pacífico Latinoamericano (APL). Esta última surge en 2006 como una propuesta del gobierno peruano para fomentar la cooperación e integración de los países de América Latina con costas bañadas por el océano Pacífico. Sin embargo, no todas las naciones latinoamericanas reciben de manera positiva lo expuesto por el miembro de la Comunidad Andina (CAN). Al respecto, los países de la Alianza Bolivariana para los pueblos de nuestra América (ALBA) consideraron que el APL era un acto de subordinación a los intereses de Estados Unidos por encima de la unión latinoamericana (Briceño, 2010). Paralelamente, otras naciones de la región se unieron al llamado de una integración regional. Debido a lo anterior, en enero de 2007 se promovió, por iniciativa del ministro de Comercio de Colombia, Jorge Humberto Botero, una reunión de ministros de Comercio en la ciudad de Cali para hablar sobre dicha integración. El resultado fue la creación de un foro sobre la iniciativa de la Cuenca del Pacífico Latinoamericano (Briceño, 2010; García, 2013).

En las posteriores reuniones derivadas de dicho foro, Chile, Colombia, México y Perú coinciden en la apertura económica y comercial. El 28 de abril de 2011 los jefes de Estado de los cuatro países determinaron establecer la Alianza del Pacífico y en 2012 se constituye jurídicamente (Briceño, 2010; García, 2013; Alianza del Pacífico, 2016).

La importancia de esta Alianza radica en lo que representan sus economías en conjunto para América Latina: 38 \% del PIB de la región, 
más de $50 \%$ de las exportaciones e importaciones de bienes, 43,5\% de la IED, así como 35,6 \% de la población total en 2014 (Alianza del Pacífico, 2016; UNCTAD, 2016). Es importante resaltar el papel de la economía mexicana en estos porcentajes: por sí sola, representa $36,6 \%$ y 35,5 \% de las exportaciones e importaciones latinoamericanas de bienes, respectivamente, así como más del $28 \%$ del PIB de la región para 2014 (UNCTAD, 2016).

Si bien la firma de la iniciativa de integración entre Chile, Colombia, México y Perú se realizó hace cinco años, es un buen momento para evaluar uno de sus objetivos en materia de comercio internacional “... avanzar progresivamente hacia la libre circulación de bienes, servicios, capitales y personas" (Alianza del Pacífico, 2016, s/p).

Debido a lo anterior, el objetivo de este documento es analizar la evolución del comercio de bienes manufacturados entre las naciones de la Alianza del Pacífico. Lo anterior para determinar el grado de avance en la integración de las economías de los países signatarios e identificar si el comercio entre estas naciones está relacionado con un nivel de ingreso per cápita similar.

Para analizar el comercio internacional entre los países de la Alianza del Pacífico es necesario especificar el tipo de producto (ya que bienes y servicios implican sectores económicos diferentes). En este estudio se consideran exclusivamente los bienes manufacturados porque son estos bienes los que pueden impulsar, efectivamente, el crecimiento económico (ONUDI, 2013). De hecho, existen algunas propuestas teóricas como las Leyes de Kaldor que consideran que la industria manufacturera es una actividad determinante en el crecimiento económico de una nación (Bernat, 1996).

Respecto de los bienes manufacturados, de acuerdo a la UNCTAD (2016), México ha disminuido las exportaciones, en términos relativos, con todos sus socios, a excepción de Perú durante el periodo 2011-2014 (ver gráfica 1). La Alianza del Pacífico representa 2,23 \% de las exportaciones mexicanas de bienes manufacturados. 


\section{Gráfica 1. Exportaciones mexicanas de bienes manufacturados a los países de la Alianza del Pacífico en términos relativos, varios años}

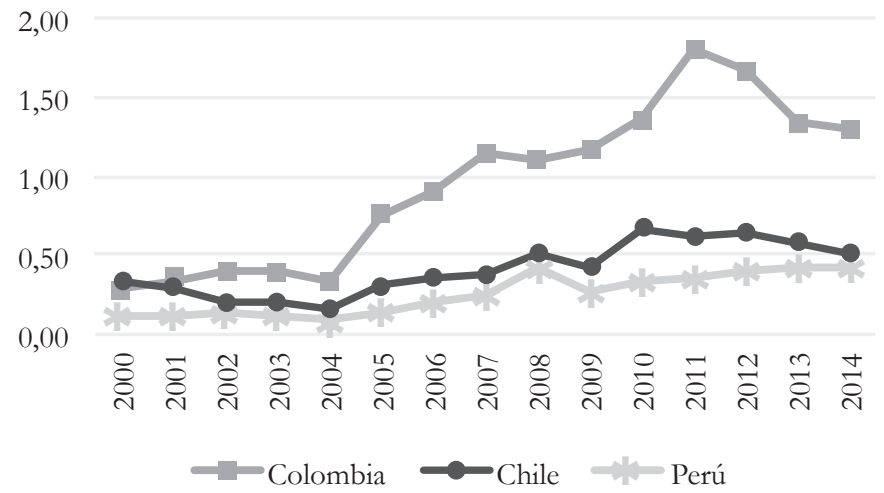

Fuente: Elaboración propia con base en UNCTAD (2016).

Los principales productos manufacturados de exportación mexicanos, en 2014, para Colombia, están representados por vehículos de motor para transporte de personas, receptores de televisión y vehículos de motor para transporte de bienes. Hacia el mercado chileno, receptores de televisión, vehículos de motor para transporte de personas y fertilizantes, y para Perú, receptores de televisión y vehículos de motor para transporte de personas (UNCTAD, 2016).

Las importaciones de los bienes manufacturados de los países de la Alianza representan, para México, 0,63\% del total de las compras que realiza del exterior (UNCTAD, 2016). Cabe destacar que, antes de la firma de la Alianza del Pacífico, las importaciones mexicanas con origen colombiano y chileno eran mayores en términos relativos. Solo Perú aumentó sus ventas a México durante el periodo 2011-2014 (ver gráfica 2).

En cuanto a Colombia, sus ventas a los países de la Alianza disminuyeron de 2011 a 2014 al representar 8,20\% y 5,62\% del total de las exportaciones de bienes manufacturados, respectivamente (UNCTAD, 2016). Destacan las ventas a México que aumentaron ligeramente durante este mismo periodo (ver gráfica 3). Cabe mencionar que las ventas colombianas a México y Perú simbolizaban un mayor porcentaje del total de sus exportaciones antes de la firma de 
la Alianza. Los principales productos de exportación de Colombia son: hacia México, vehículos de motor para transporte de personas y carbón pulverizado; para Chile, carbón pulverizado, aceites de petróleo y alquitrán, y hacia Perú, aceites de petróleo y alquitrán, así como, perfumería, cosméticos, papel higiénico (UNCTAD, 2016).

\section{Gráfica 2. Importaciones mexicanas de bienes manufacturados de los países de la Alianza del Pacífico en términos relativos, varios años}

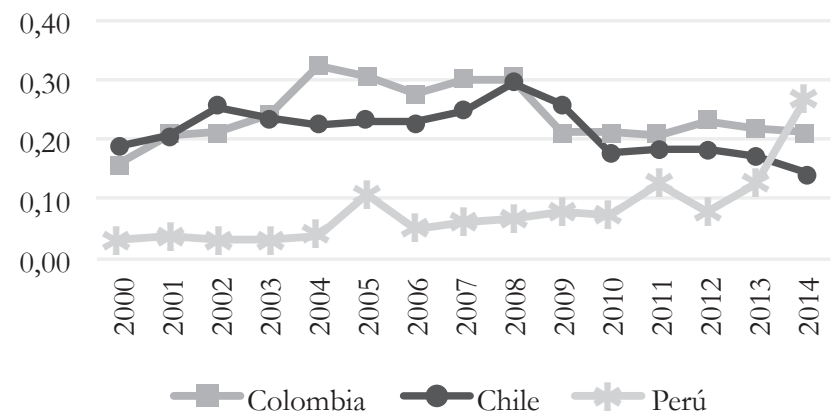

Fuente: Elaboración propia con base en UNCTAD (2016).

Gráfica 3. Exportaciones colombianas de bienes manufacturados a los países de la Alianza del Pacífico en términos relativos, varios años

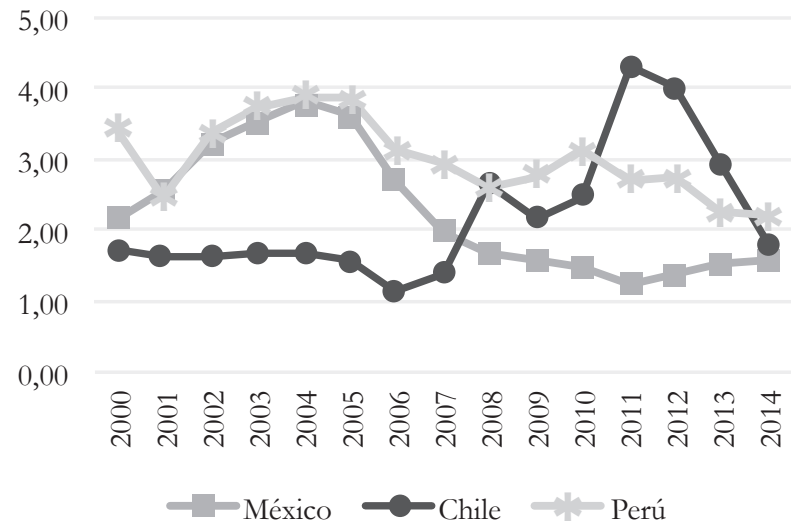

Fuente: Elaboración propia con base en UNCTAD (2016).

Respecto de las importaciones colombianas de bienes manufacturados, se han mantenido constantes en términos relativos respecto 
a Chile y Perú. Relativo a México, se puede apreciar que es el país socio a quien más compras de bienes manufacturados realiza, aunque la participación porcentual de las importaciones mexicanas en la economía colombiana ha disminuido a partir del año en que se formaliza la Alianza del Pacífico (ver gráfica 4). De manera conjunta, las importaciones colombianas de los países de la Alianza han disminuido su participación porcentual de 2011 a 2014 al pasar de 14,23 \% a 11,03\%, respectivamente (UNCTAD, 2016).

\section{Gráfica 4. Importaciones colombianas de bienes manufacturados de los países de la Alianza del Pacífico en términos relativos, varios años}

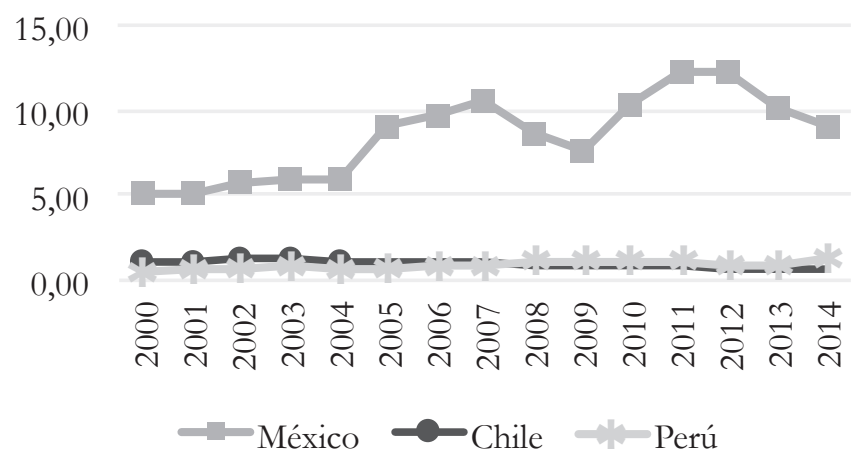

Fuente: Elaboración propia con base en UNCTAD (2016).

En lo que respecta a Chile, el país con quien logra mayor comercio en términos de exportaciones es Perú, ya que este último constituye más de $10 \%$ de las ventas chilenas de bienes manufacturados al mundo (ver gráfica 5). Los países de la Alianza, en su conjunto, representan más de $20 \%$ de las exportaciones manufactureras chilenas, manteniéndose más o menos estable de 2011 a 2014 (UNCTAD, 2016). Los principales productos chilenos de exportación a sus socios comerciales son: para México y Colombia, chapas y productos de madera, fertilizantes y papel, y hacia Perú, fertilizantes y papel (UNCTAD, 2016). 
184 / Wendy Ovando Aldana, Rosa Azalea Canales García, Gabriela Munguía VázQuez

Gráfica 5. Exportaciones chilenas de bienes manufacturados a los países de la Alianza del Pacífico en términos relativos, varios años

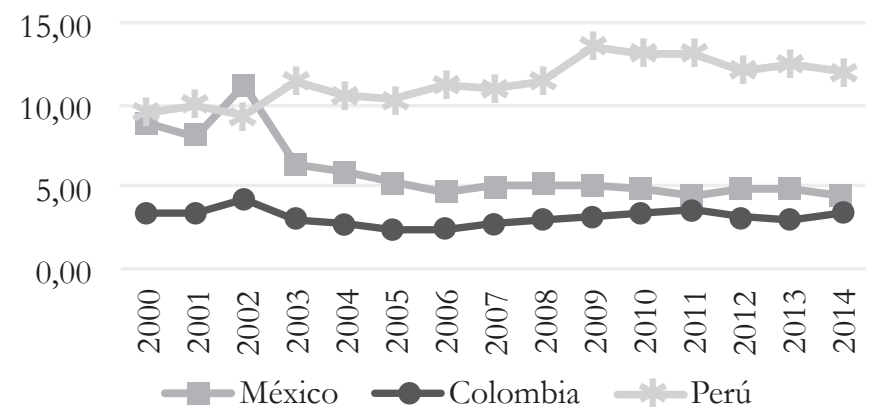

Fuente: Elaboración propia con base en UNCTAD (2016).

Respecto de las importaciones chilenas de bienes manufacturados, de los países de la Alianza, México es su principal socio. Si bien ha aumentado la participación de las importaciones de productos mexicanos en términos relativos, no se ha alcanzado el nivel del año 2000, periodo previo a la firma de la iniciativa de integración regional (ver gráfica 6). Los tres países socios de Chile han disminuido su participación dentro de las compras que realizan los chilenos al exterior: de 7,29 \% en 2011 pasaron a 5,60 \% en 2014. Lo anterior se debió al descenso representativo de las compras a Colombia, principalmente.

Gráfica 6. Importaciones chilenas de bienes manufacturados de los países de la Alianza del Pacífico en términos relativos, varios años

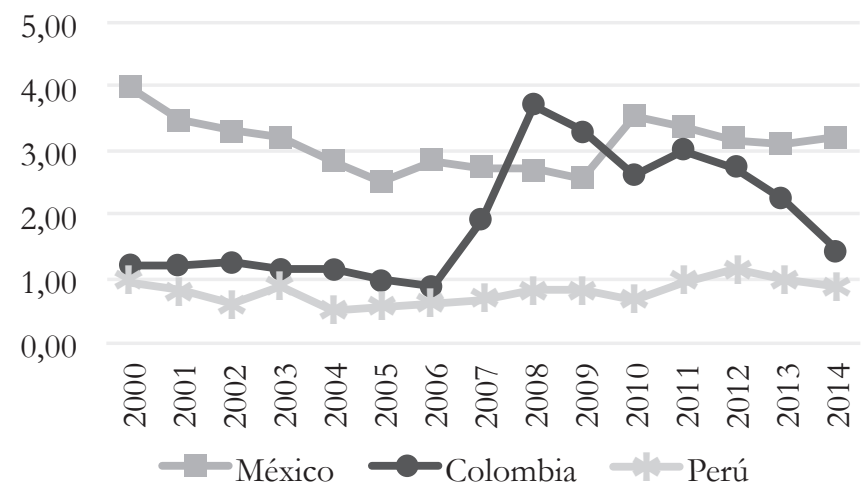

Fuente: Elaboración propia con base en UNCTAD (2016). 
Finalmente, en cuanto a las exportaciones peruanas de bienes manufacturados hacia sus socios, estas han aumentado, en términos relativos, principalmente a Colombia y México. Destaca su relación comercial con Chile, ya que era mayor la participación de las compras de este tipo de bienes a los chilenos previo a la firma de la Alianza (ver gráfica 7). Los principales productos manufacturados que vende Perú a los miembros de la Alianza del Pacífico son: hacia México, gas natural y aceites de petróleo o de mineral bituminoso; para Colombia, aceites de petróleo o de mineral bituminoso y, placas, hojas, películas, bandas y láminas, de plástico; y hacia Chile, aceites de petróleo y alquitrán, así como elementos químicos inorgánicos, óxidos y sales de halógenos (UNCTAD, 2016).

\section{Gráfica 7. Exportaciones peruanas de bienes manufacturados a los países de la Alianza del Pacífico en términos relativos, varios años}

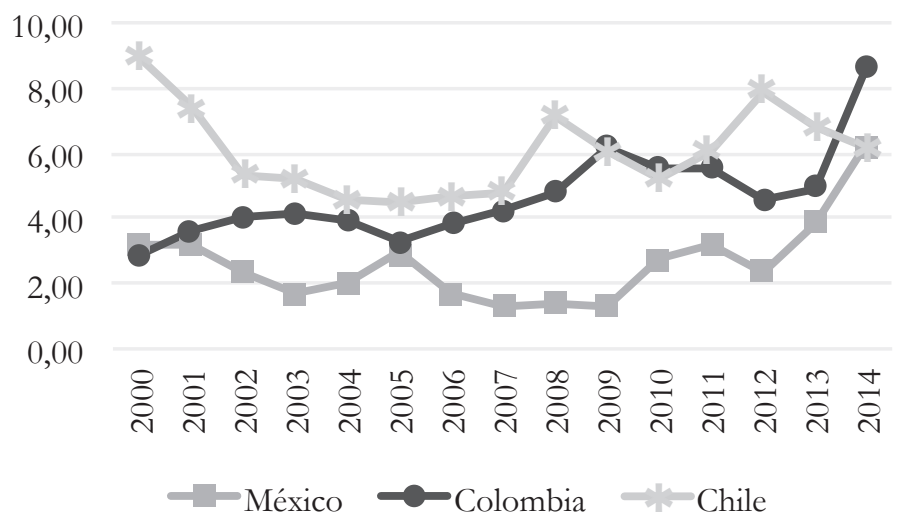

Fuente: Elaboración propia con base en UNCTAD (2016).

Respecto de las compras de bienes manufacturados que Perú realiza a sus socios comerciales, se han mantenido estables en promedio: la Alianza del Pacífico representaba 10,54 \% y 9,81 \% de las importaciones peruanas totales en 2011 y 2014, respectivamente (UNCTAD, 2016). Lo anterior se debe a que, si bien Chile y Colombia disminuyeron su participación, las compras de bienes manufacturados mexicanos aumentaron en términos relativos en este periodo de tiempo (ver gráfica 8). 


\section{Gráfica 8. Importaciones peruanas de bienes manufacturados de los países de la Alianza del Pacífico en términos relativos, varios años}

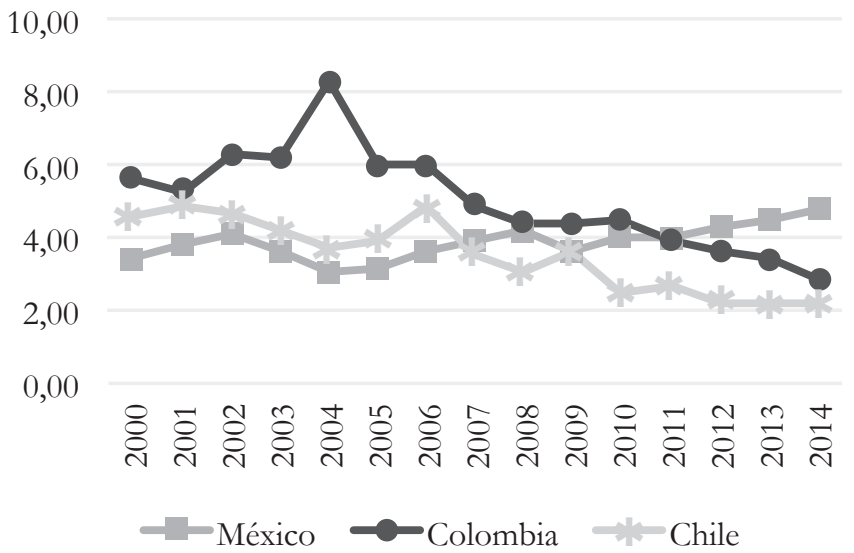

Fuente: Elaboración propia con base en UNCTAD (2016).

De los datos presentados en esta sección, se infiere que México tiene una mayor relación comercial con Colombia en términos de exportaciones e importaciones, su vinculación con Chile tiende a disminuir, pero con Perú tiende a aumentar. Aun así, para México, las exportaciones e importaciones de bienes manufacturados, hacia y desde los países de la Alianza del Pacífico, en 2014, representan $2,23 \%$ y $0,63 \%$, respectivamente. Las exportaciones mexicanas de bienes (materias primas y manufacturados) están más asociadas a la economía de Estados Unidos, ya que estas representan 80,2\% de las exportaciones totales, y $49 \%$ de las compras que realizan los mexicanos al mundo provienen del país vecino del norte (OMC, 2015). Así, la Alianza del Pacífico no es representativa para el comercio internacional mexicano, en términos relativos.

En cuanto a los países sudamericanos, el conjunto presenta una balanza comercial de bienes manufacturados deficitaria con México. Colombia y Chile ostentan mayor relación comercial con México en las importaciones. Ambos países adquieren productos mexicanos con mayor valor agregado (vehículos de motor). Sin embargo, en cuanto a las exportaciones, Colombia comercializa mayor porcentaje con Chile; 
y Chile, con Perú. Por su parte, Perú destaca por exhibir aumentos en las exportaciones e importaciones con la economía mexicana, a pesar de la distancia que implica realizar comercio con México.

En términos relativos, la Alianza del Pacífico simboliza para Colombia $5,62 \%$ y $11,03 \%$ de sus exportaciones e importaciones de bienes manufactureros, respectivamente. No obstante, Estados Unidos y la Unión Europea configuran el principal destino del comercio de bienes colombianos: $26,4 \%$ y 17,2\%. En relación al origen de los bienes que consumen los colombianos, Estados Unidos (28,5 \%) y China $(18,4 \%)$ (OMC, 2015) son más representativos para la economía colombiana que los países de la Alianza, a pesar de que existe menor distancia con Chile y Perú.

Relativo a Chile, su comercio está más asociado con China: exporta hacia esta nación asiática 24,6 \% del total de bienes y les compra 20,9\% de las importaciones totales. La Unión Europea y Estados Unidos representan, de manera conjunta, 26,7 \% de las exportaciones totales de los bienes producidos en Chile y $34,2 \%$ de las importaciones totales que realizan los chilenos (OMC, 2015). La Alianza del Pacífico representa para Chile, en 2014, 20,12\% de las exportaciones de bienes manufacturados y 5,6 \% de las importaciones de los mismos bienes.

Finalmente, Perú es la economía para quien la Alianza del Pacífico, respecto de bienes manufacturados, representa mayor comercio en términos relativos en comparación con el resto de los miembros: exportaciones, 21,01\% e importaciones, 9,81\%, en 2014. Sin embargo, al igual que México, Colombia y Chile, sus exportaciones se dirigen en mayor porcentaje a otros países. De acuerdo a la OMC (2015) China representa el principal destino y origen de las exportaciones e importaciones de los bienes peruanos con 18,3\% y 21,2\%, respectivamente. Estados Unidos y la Unión Europea, de manera conjunta, conforman $32,8 \%$ de las exportaciones totales de los bienes producidos en Perú y $32,6 \%$ de las importaciones totales que realizan los peruanos.

La Alianza del Pacífico representa un mercado para los bienes producidos en cada una de sus naciones, aunque existen otros mercados 
de mayor interés con quiénes comerciar. Una forma de confirmar lo anterior es a través del modelo gravitacional, el cual se describe en el siguiente apartado, así como su modificación para relacionarlo con la Teoría de Linder.

\section{El modelo gravitacional en los países de la Alianza del Pacífico}

El modelo gravitacional se ha empleado para verificar la Teoría de Linder. En esta sección se describe su especificación y modificación para la hipótesis principal de la teoría ya mencionada.

\section{El modelo gravitacional}

La forma original del modelo gravitacional se deriva de la Ley de la Gravedad de Newton (Malik y Mir, 2014) y, en términos económicos, expresa que el volumen del comercio entre diferentes economías es directamente proporcional al tamaño del ingreso de las naciones, pero inversamente proporcional a la distancia que existe entre estos espacios económicos (López y Muñoz, 2008). El modelo en cuestión se centra en la interacción entre la resistencia (distancia geográfica) y la atracción (patrones similares de demanda) (Appleyard, Field y Cobb, 2010).

La forma original del modelo gravitacional se expresa de la siguiente manera:

$$
E X P_{i j t}=\alpha \frac{G D P_{i t} G D P_{j t}}{\text { Distance }_{i j}}
$$

Donde $\mathrm{EXP}_{\mathrm{ijt}}$ es el volumen del comercio ${ }^{1}$ (exportaciones) del país $i$ al país $j$ en el periodo $t ;$ GDP $_{\mathrm{it}}$ así como GDP ${ }_{\mathrm{jt}}$ representan el producto nacional de los países $i$ y $j$ respectivamente; DIST $\mathrm{T}_{\mathrm{ij}}$ es la distancia

\footnotetext{
1 En Malik y Mir (2014), la variable volumen del comercio aparece como TRADE. Se ha sustituido por EXP para estar en concordancia con la ecuación de Mátyás (1997) puesto que es la especificación correcta del modelo gravitacional.
} 
entre los países $i$ y $j$; $\alpha$ es la constante de proporcionalidad (Mátyás, 1997; Malik y Mir, 2014).

Al aplicar logaritmos a la ecuación 1, se transforma en una función lineal. La especificación econométrica correcta del modelo gravitacional (Mátyás, 1997) es la siguiente:

$E X P_{i j t}=\beta_{0}+\beta_{1} \operatorname{In} G D P_{i t}+\beta_{2} \operatorname{In} G D P_{j t}+\operatorname{InDist}_{j t}+\ldots+u_{j t}$

Donde $u_{i j}$ representa el error estocástico.

Importante mencionar que para probar la hipótesis de Linder, "el comercio internacional de bienes manufacturados será más intensivo entre los países con un ingreso per cápita similar, en comparación con los países que cuentan con un nivel de ingreso per cápita diferente" (Appleyard, Field y Cobb, 2010), es necesario realizar algunos ajustes a la ecuación 2 como se muestra en la adaptación del modelo gravitacional a la Teoría de Linder.

\section{El modelo gravitacional en la Teoría de Linder}

Si bien Linder no especificó un modelo formal para su hipótesis (Bukari et al., 2005) algunos trabajos empíricos han empleado el modelo gravitacional con ligeras modificaciones (Hallak, 2010). Es necesario subrayar que los países con ingreso per cápita similar tienden a estar más cercanos geográficamente, por lo tanto, los costos de transporte no son controlados entre estos países, así que su comercio internacional puede asociarse más a los bajos costos de transporte que a la similitud en sus ingresos; de ahí que el modelo gravitacional sufra algunas modificaciones para probar la hipótesis de Linder (Hallak, 2010). Aunque existen diversas versiones empíricas sobre la especificación del modelo para la Teoría de Linder (Choi, 2002; Bukari et al., 2005; Hallak, 2010) la ecuación para este estudio se retoma de Hallak (2010):

$\ln I M P \ddot{j j t}=\beta_{0}+\beta_{1} \ln G D P_{i t}+\beta_{2} \ln G D P_{j t}+\beta_{3} \ln D i s t i j+\beta_{4} \ln L I N D E R_{i j}+u_{i j}$ 
La Teoría de Linder especifica que, generalmente, los países en desarrollo exportan más bienes primarios que manufacturados. Por lo tanto, las exportaciones (EXP) que aparecían en la ecuación 2 se sustituyen por importaciones (IMP); ya que las compras de los países en desarrollo están más asociadas a los bienes industriales y permiten, entonces, probar a la teoría (McPherson, Redfearn y Tieslau, 2001; Bukari et al., 2005).

En la ecuación 3, la variable dependiente, IMP, mide la intensidad del comercio entre los países $i$ y $j$. Esta estará en función de cuatro variables exógenas o independientes: producto nacional del país $i$ en el periodo $t\left(G P_{i t}\right)$, producto nacional del país $j$ en el periodo $t$ (GDP.), distancia geográfica entre los países $i$ y $j$ (Dist), y el término LINDER. Se espera que exista una relación positiva entre dos variables explicativas y la variable endógena: $\mathrm{GDP}_{\mathrm{it}}$ y $\mathrm{GDP}_{\mathrm{jt}}$. En otras palabras, se supone que a mayor producción nacional, tanto del país de origen como del país con el que comercia, mayor será la intensidad del comercio. No obstante, a mayor distancia, menor comercio entre los países $i$ y $j$ (McPherson, Redfearn y Tieslau, 2001; Hallak, 2010). Lo mismo ocurre con el término LINDER; sin embargo, es primordial, dentro de las hipótesis del modelo, discutir sobre este término representado por las diferencias del ingreso per cápita entre las naciones de estudio. Se supone que este debe ser un término menor cuando la disimilitud en el ingreso entre los países es también menor, por lo tanto, la predicción de la hipótesis de Linder es que el coeficiente $\beta_{4}$, de la ecuación 3, será menor a cero (Hallak, 2010). Lo anterior significa que si la diferencia en el ingreso per cápita de dos países es mínima, el comercio entre estas naciones se intensifica.

\section{Datos}

Para cuantificar las variables se emplearon las bases de datos de la UNCTAD (2016). En específico, para la variable IMP (variable endógena) se obtuvieron las importaciones de bienes de cada uno de los países signatarios de la Alianza del Pacífico, desde 1995 hasta 2014, con el fin de contar con mayor número de datos. La UNCTAD presenta tanto bienes primarios como secundarios de manera conjunta, 
así que se depuraron las bases conforme a la clasificación que presenta dicho organismo (UNCTAD product groupings and composition). De esta manera se incluyeron exclusivamente los bienes del sector secundario. Cabe resaltar que los datos se hallan en miles de dólares a precios constantes de 2005 .

Para las variables GDP it $_{\text {y }}$ GDP $_{\mathrm{j}}$ se empleó el producto interno bruto total (gross domestic product) en millones de dólares, también a precios de 2005. Las distancias se referencian considerando la información de la página http://es.distance.to/ que permite obtener la longitud entre las ciudades capitales de cada país. Finalmente, para el término de LINDER, se utilizaron los productos nacionales per cápita que proporciona la base de datos de la UNCTAD por país y se calcularon las diferencias absolutas como lo recomiendan otros estudios (McPherson, Redfearn y Tieslau, 2001; Bukari et al., 2005). Finalmente, se obtuvo el logaritmo natural de los datos seleccionados.

Se empleó como software estadístico STATA 12 por tratarse de datos de panel. En la siguiente sección se muestran los resultados.

\section{Resultados}

Los resultados empíricos se obtuvieron empleando dos marcos teóricos diferentes para datos de panel: el modelo de efectos fijos y el modelo de efectos aleatorios (Jiménez y Narbona, 2010). Aplicando el test de Hausman en el programa STATA 12, se comprobó que el modelo de efectos fijos era idóneo para este estudio.

Como se puede observar en el cuadro 1, la variable distancia no se presenta en los resultados debido a que el modelo de efectos fijos absorbe variables independientes que no varían en el tiempo como la distancia y las variables dummy (Cheng y Wall, 2005; Vemury y Siddiqi, 2009). 
192 I Wendy Ovando Aldana, Rosa Azalea Canales García, Gabriela Munguía VázQuez

Cuadro 1. Estimación del modelo de efectos fijos sobre las importaciones

\begin{tabular}{|l|c|c|c|}
\hline \multicolumn{1}{|c|}{ Países } & GDPi & GDPj & LINDER \\
\hline México & 1.540913 & .8399302 & -.112834 \\
& $(0.090)$ & $(0.153)$ & $(0.287)$ \\
\hline Colombia & 1.781342 & .7165596 & -.2054449 \\
& $\mathbf{( 0 . 0 0 5 )}$ & $(0.208)$ & $(0.156)$ \\
\hline Chile & .5523439 & 1.54322 & .154818 \\
& $(0.377)$ & $\mathbf{( 0 . 0 2 1 )}$ & $(0.085)$ \\
\hline Perú & 2.165709 & -1.120649 & .4329837 \\
& $\mathbf{( 0 . 0 0 0 )}$ & $\mathbf{( 0 . 0 4 2 )}$ & $\mathbf{( 0 . 0 0 0 )}$ \\
\hline
\end{tabular}

Nota: Se obtuvieron 60 observaciones para cada país. El nivel de significancia de los coeficientes se presenta en paréntesis.

Para el caso de México, ninguna de las variables es estadísticamente significativa. Colombia y Chile presentan dos coeficientes estadísticamente significativos (menores a 0.05 ): el producto nacional y el producto nacional de los países socios, respectivamente. Es decir, la producción de Colombia tiene un efecto positivo en sus importaciones: por cada aumento de $1 \%$ en la producción nacional colombiana, las importaciones aumentan 1,78\%. Para Chile se observa que el tamaño de la economía de las naciones de la Alianza del Pacífico tiene un impacto significativo en las importaciones chilenas: por cada aumento de $1 \%$ en la producción nacional de los socios de la Alianza, las importaciones chilenas aumentan 1,54 \%. En el caso de Perú, todas las variables del modelo son estadísticamente significativas, aunque la producción de sus países socios y el término LINDER presentaron signos inversos a los esperados, por lo que es preciso realizar la interpretación de estos cautelosamente.

Los resultados obtenidos proveen un débil soporte a la hipótesis de Linder: "el comercio internacional de bienes manufacturados será más intensivo entre los países con un ingreso per cápita similar, en comparación con los países que cuentan con un nivel de ingreso per cápita diferente". 


\section{Conclusiones}

A cinco años de la firma de la Alianza del Pacífico, el comercio entre México, Colombia, Chile y Perú es escaso; aunque hay signos de aumento en las relaciones comerciales, en términos relativos, entre Perú y México y Perú y Colombia. Estos resultados coinciden con los hallazgos derivados del modelo gravitacional: hay evidencia estadística débil para identificar una fuerte relación comercial entre los países de la Alianza a pesar de contar con niveles de ingreso per cápita similares. Por lo tanto, el avance hacia la integración de las economías de esta Alianza, desde el punto de vista comercial, es casi nulo.

Los hallazgos de esta investigación coinciden con Hernández y Muñoz (2015) quienes identificaron un comercio marginal entre estas naciones. No obstante, la relevancia del estudio presentado en líneas precedentes versa en que propone un esquema novedoso basado en técnicas de análisis regional aplicadas a contextos de comercio internacional, en particular, el relativo a la Alianza del Pacífico.

De igual forma, la evidencia estadística permite establecer que el comercio internacional de bienes manufacturados de la Alianza del Pacífico no es representativo, en términos relativos, principalmente para México. De hecho, la economía mexicana está más asociada a la de Estados Unidos. Las exportaciones de bienes mexicanos tienen como principal destino al país vecino del norte. Lo anterior coincide con Morales, Almonte y Carbajal (2012) quienes, al estudiar el comercio entre México y Uruguay, concluyen que este resulta insignificante por la gran influencia que tiene el PIB de Estados Unidos sobre el PIB de México. Es más, existe evidencia empírica sobre la integración de la economía mexicana a la de Estados Unidos, lo cual ha derivado en una sincronización de los ciclos económicos de ambos países (Mejía, Gutiérrez y Farías, 2006).

La Alianza del Pacífico representa 2,23 \% y 0,63\% de las exportaciones e importaciones mexicanas de bienes manufacturados. Es decir, el comercio mexicano con Colombia, Chile y Perú es mínimo en conjunto. 
En cuanto a Colombia ocurre algo similar: su mayor socio comercial es Estados Unidos a pesar de la distancia relativamente corta con Chile y Perú, o bien, de la mayor cercanía con la economía mexicana respecto de la estadounidense. La Alianza del Pacífico representa, para Colombia, 5,62\% y 11,03 \% de sus exportaciones e importaciones de bienes manufactureros, cifras por debajo de lo que vende o compra Colombia a Estados Unidos: 26,4 \% y 28,5 \%, respectivamente.

Destaca el hecho de que las economías chilena y peruana tienen una mayor participación en el comercio de bienes manufacturados con los países miembros de la Alianza del Pacífico: alrededor del $20 \%$ de sus exportaciones se realiza con esta iniciativa de integración. No obstante, ambos países muestran mayor comercio con China.

Respecto a las limitantes de esta investigación, es factible señalar que la Teoría de Linder no pudo ser sustentada con los datos estadísticos presentados, por lo que se recomienda tener cuidado en las conclusiones puesto que sería idóneo ampliar la muestra a un periodo mucho más largo. De igual forma, este estudio solo contempló el comercio de bienes manufacturados, por lo que analizar los sectores primario y terciario se podría considerar como otros temas de investigación.

En síntesis, el estudio de la Alianza del Pacífico permite a investigadores, académicos, políticos y a la sociedad en general, analizar la viabilidad de este y otros proyectos de integración económica puesto que plantean objetivos ambiciosos como lograr el crecimiento y desarrollo económico cuando el comercio entre los países que los conforman es marginal.

\section{Referencias}

Alianza del Pacífico (2016). Recuperado de https://alianzapacifico.net/ que-es-la-alianza/\#la-alianza-del-pacifico-y-sus-objetivos

Antunes, R. (2005). Los sentidos del trabajo. Argentina: Ediciones Herramientas y Taller de Estudios Laborales. 
Appleyard, D. R., Field, A. J. \& Cobb, S. L. (2010). International Economics. New York: McGraw-Hill.

Arnon, A. \& Weinblatt, J. (1998). Linder's Hypothesis Revisited: Income Similarity Effects for Low income Countries. Applied Economic Letters, 5, 607-611.

Bernat, G. A. (1996). Does Manufacturing Matter? A Spatial Econometric View of Kaldor's Laws. Journal of Regfional Science, 36 (3). 463-477.

Brenner, R. (2009). La economía de la turbulencia global. Madrid: Akal.

Briceño, J. L. (2010). La iniciativa del Arco Pacífico latinoamericano. Un nuevo actor en el escenario de la integración regional. Nueva Sociedad, (228), 44-59.

Bukhari, S. S., Ahmad, M. H., Alam, S., Bukhari, S. S. \& Butt, M. S. (2005). An Empirical Analysis of the Linder Theory of International Trade for South Asian Countries. Pakistan Development Review, (3), 307.

Carbaugh, R. J. (2004). Economía internacional. México: Thomson.

Castillo, D. (2009). Los nuevos trabajadores precarios. México: Miguel Ángel Porrúa.

Choi, C. (2002). Linder Hypothesis Revisited. Applied Economics Letters, 9, 601-605, Business Source Complete, EBSCO host, viewed 14 May 2016.

Chow, P., Kellman, M., \& Shachmurove, Y. (1999). A Test of the Linder Hypothesis in Pacific NIC Trade 1965-1990. Applied Economics, 31 (2), 175-182. doi: 10.1080/000368499324408

Duarte, L. K., González, C. H. \& Montoya, D. A. (2014). Colombia de cara al nuevo regionalismo renovado: la Alianza del Pacífico. Revista Punto De Vista, 5 (9), 139-161.

Ekelund, R. B. \& Hébert, R. F. (2005). Historia de la teoría económica y su método. México: McGraw-Hill.

Falomir, N. (2013). La identidad de UNASUR: ¿regionalismo post-neoliberal o post-hegemónico? Revista de Ciencias Sociales (Cr), II, 97-109. Recuperado de http:/ /www.redalyc.org/articulo.oa?id=15329874007 García, J. (2013). Alianza del Pacífico. ¿Hacia dónde vamos? Agenda Internacional, 20 (31), 43-54.

Hallak, J. C. (2010). A Product-Quality View of the Linder Hypothesis. The Review of Economics and Statistics, XCII (3), 453-466.

Harvey, D. (1998). La condición de la posmodernidad. Investigación sobre los orígenes del cambio cultural. Argentina: Amorrortu Editores.

Harvey, D. (2007). Breve historia del neoliberalismo. Madrid: Akal. 
Jiménez, J. C. \& Narbona, A. (2010). Los factores institucionales como determinantes de los flujos comerciales internacionales. Revista de Economia Mundial, (24), 23-48.

López, D. \& Muñoz, F. A. (2008). Los modelos de gravedad en América Latina: el caso de Chile y México. Comercio Exterior, 58(11), 803-813.

Malik, I. A. \& Mir, M. A. (2014). India’s Trade Potential with Central Asia: An Application of Gravity Model Analysis. International Journal On World Peace, (3), 53-69.

Martínez, R. \& Soto, E. (2012). El Consenso de Washington: la instauración de las políticas neoliberales en América Latina. Política y Cultura, (37), 35-64.

Mcpherson, M. A., Redfearn, M. R. \& Tieslau, M. A. (2001). International Trade and Developing Countries: An Empirical Investigation of the Linder Hypothesis. Applied Economics, 33(5), 649-657. doi:10.1080/000368401750106243

Mejía, P., Gutiérrez, E. E. \& Farías, C. A. (2006). La sincronización de los ciclos económicos de México y Estados Unidos. Investigación Económica, LXV (258), 15-45.

Morales, M. E., Almonte, L. \& Carbajal, Y. (2012). A ocho años del TLC México-Uruguay. Una evaluación. Estudios Internacionales, (172), 7-29. Organización de las Naciones Unidas para el Desarrollo Industrial (ONUDI). Informe sobre el Desarrollo Industrial 2013. En línea: file:// C:/ Users/titulacion/Downloads/Desarrollo_Industrial_2013.pdf

Organización Mundial del Comercio (OMC). Perfiles Comerciales 2015. Recuperado de https://www.wto.org/spanish/res_s/booksp_s/trade_profiles15_s.pdf

Raffo, L. (2012). Una reconstrucción milliana del modelo ricardiano de comercio internacional. Cuadernos de Economía, XXXI (56) 21-57. Recuperado de http://www.redalyc.org/articulo.oa?id=282124593002 Sanahuja, J. A. (2007). Regionalismo e integración en América Latina: balance y perspectivas. Pensamiento Iberoamericano, 75-106.

Sánchez, M. (1999). Efectos económicos de acuerdos regionales de comercio: una revisión de argumentos. Buenos Aires: CEPAL.

Solís, L. (2000). La realidad económica mexicana: retrovisión y perspectivas. México: Fondo de Cultura Económica. 
Tan, L. \& Zeng, D. (2014). Spatial Inequality Between Developed and Developing Economies. Papers In Regional Science, 93(2), 229-248. doi:10.1111/pirs.12046

United Nations Conference on Trade and Development (UNCTAD) (2016). Statistics. Recuperado de http://unctad.org/en/Pages/Statistics.aspx

Van Klaveren, A. (1997). América Latina: hacia un regionalismo abierto. Estudios Internacionales, 30(117), 62-78.

Whiteside, N. (2000). From Full Employment to Flexibility: Britain and France in Comparison, 1960-2000. Chapter 3. En Strath, Bo. (Ed.), After Full Employment. European Discourses on Work and Flexibility (pp. 107-133). Brussels: PIE-Peter Lang. 